\title{
Early mobilization in LVAD recipients: An obligatory step towards recovery
}

\author{
Ugo Corrà ${ }^{1}$, Massimo Pistono 2 \\ ${ }^{1}$ Exercise Pathophysiology Laboratory, Istituti Clinici Scientifici Maugeri, Institute of Veruno; ${ }^{2}$ Laboratory for the \\ analysis of Cardio-Respiratory Signals, Istituti Clinici Scientifici Maugeri, Institute of Veruno (NO), Italy
}

Chronic heart failure (CHF) is a devastating clinical syndrome [1]. Therapeutic schemes for have developed rapidly from conventional medical therapy to biomedical strategies, interventional, mechanical, that incorporates left ventricular assistant device (LVAD), surgical, regenerate, and rehabilitating policies. These therapies have improved the symptoms and life expectancy [2].

Early-generation LVAD were complex, large devices that provided short-term support, were prone to malfunction and were associated with significant adverse events: newer generation LVADs with simpler design principles, enhanced durability, and better hemocompatibility have improved clinical outcomes [3]. As a result, there has been greater acceptance of VAD therapy as a viable option for patients with advanced heart failure.

Nevertheless, timing of implantation is essential: the distance from implantation forces medical strategies.

\section{From 0 to 10-15 days}

Placement of a left ventricular assistant device (LVAD) is a serious surgical procedure [4]: many placements are done in patients who have had prior cardiac surgical procedures, and, moreover, since most of the potential recipients are often hemodynamically unstable, institution of cardiopulmonary bypass is required. All issues of safe sternal division and heart chamber dissection and mobilization that apply to standard cardiac reoperations apply to LVAD operations [4], together with restoration of hemodynamic stability and prevention of death. Recovery time after LVAD sur-

\footnotetext{
Correspondence: Ugo Corrà, Head of the Exercise Pathophysiology Laboratory, Istituti Clinici Scientifici Maugeri, Veruno Institute, Via per Revislate 13, 28010 Veruno (NO), Italy.

Tel. +39.0322.884711 - Fax: +39.0322.830294.

E-mail: ugo.corra@icsmaugeri.it

Key words: Chronic heart failure; LVAD placement; cardiac surgery.

Received for publication: 26 February 2019.

Accepted for publication: 1 March 2019

(C) Copyright U. Corrà and M. Pistono, 2019

Licensee PAGEPress, Italy

Monaldi Archives for Chest Disease 2019; 89:1056

doi: 10.4081/monaldi.2019.1056
}

This article is distributed under the terms of the Creative Commons Attribution Noncommercial License (by-nc 4.0) which permits any noncommercial use, distribution, and reproduction in any medium, provided the original author(s) and source are credited. gery depends on condition before the surgery: if severe heart disease before getting the LVAD was present, the body may be weak and lungs may not work very well. Thus, the patient may need a ventilator for several days after surgery. The recipient may also need to continue getting nutrition through a feeding tube. It is also important to watch for signs of infection, especially around the area where the tubes or cable from the LVAD exit the skin. Signs of infection may include soreness over the VAD site, fluid draining from the site where the tubes or cable exit the skin, or fever. The patient might need local or general antibiotics. With LVADs, anticlotting medicines may help prevent blood clots from forming in heart or the LVAD, and periodic blood tests to make sure the medicines are working properly. These medical strategies can adversely influence quality of life and functional recovery of recipients.

LVAD recipients are usually referred to physical therapy within 48 hours after surgery [5], or as early as recipient is completely awake. The early recovery phase consists of the treatment and/or prevention of pulmonary complications and improvement of respiratory function. Wound care play is key, and early mobilization (EM) is initiated to prevent complications of bedrest and minimize loss of mobility. As mentioned LVAD patient's outline is, sometimes, multifaceted, characterized by the presence of the SwanGanz catheter, infusion lines, LVAD and vital parameters monitoring, urinary catheter, and oxygen support systems, an individualized treatment plan is recommended: for LVAD recipients with concomitant acute or chronic medical problems, physical therapy goals and treatment plan should be modified [5].

\section{From 10-15 to 30-35 days}

In-patient cardiac rehabilitation is recommended in LVAD, according the patient's condition and response to physical therapy [6,7]. At this stage, monitoring of the pulmonary function still remains an important aspect in LVAD recipients; continuing evaluation of the chest imaging, together with the clinical observation, should be adopted to avoid worsening of the respiratory function. Pleural effusion, atelectasis, and lung dysventilation must be also prevented/treated by means of a respiratory program focused on respiratory exercises. Respiratory exercises can be scheduled during the day in more than one session. Upper body exercises should still be delayed to ensure sternal healing.

Since the degree of patient's mobility normally increases, there is also the need to plan a specific device training activity in order to preserve and guarantee the patient's safety. EM should be centred on patient's autonomy. Medical professionals may be hesitant to start mobilisation because of the presence of the device as specific skill and expertise are required. The health care professionals should be familiar not only with exercise physiology and the different exercise modalities but also with the functioning of the device, in order 
to face promptly all potential complications. Full patients' history, clinical and functional evaluations are prerequisites as well as heart rate monitoring: monitor exercise session for detection (and treatment) of exercise-induced arrhythmias is crucial, as well. Vital signs, self-reported symptom scores, and LVAD function should be monitored: in particular, the mean arterial pressure because hypertension would affect the LVAD capacity to pump blood forward. Hypotension and VAD blood flow alterations might be related to under-filling of the left ventricular (LV) secondary to high pump speed, right ventricular (RV) failure, arrhythmias, etc. The LVAD coordinator should be consulted if the mean pressure is below 70 $\mathrm{mm} \mathrm{Hg}$ or higher than $90 \mathrm{~mm} \mathrm{Hg}$, especially when accompanied by VAD alarm activation. Finally, the physical therapist is responsible for putting in security the cannulas, drivelines and the LVAD external equipment, to prevent damage during mobility.

Initial postural passages should be performed, beginning with the maintenance of a sitting position at the edge of the bed, progressing toward the sitting position in a chair. Then, the patient can start a more intensive muscular program oriented to develop the ability to gain autonomously an upright position. When postural passages, upright position, and in-bed movements are carried out autonomously, and patient shows the ability to carry and to manage the VAD, batteries, and controller, EM can start [4-6]. As in every patient, EM is defined as initiating physical exercise within the early illness phase. This preliminary phase is not standardised, since it is conditioned by the patient's status, facilities and referrals. Beside patient's needs, EM should be adapted: supervision from family members and/or nursing staff is warmly requested, to monitor LVAD and clinical parameters This phase is important to rule out contraindications to exercise [6] and should start only when device troublesome accounts are mostly over [5-9]. EM prevents complications of muscle deconditioning and cachexia, and, through a broad range of activities, facilitates independence; as the device is pre- and postload dependent EM might help the recovery phase.

EM favours ambulation and includes functional strengthening, muscle endurance and aerobic training, similarly to all other HF patients. Changes in gait are possible as result of earlier fatigue, appearance of new symptoms or unexpected VAD/clinical parameters changes. Finally, the duration of EM should be individualised.

\section{From 30-35 to 60-65 days}

Out-patient in or out-hospital rehabilitation can be suggested, at this stage [6,7], according to the individual's progresses and facilities. Walking, cycling, climbing stairs, and other exercises should be supervised: flexibility exercises are encouraged and tailored on patient characteristics. Physical activity can be made in small groups and the duration can range between 5 and $30 \mathrm{~min}$. Group activities are important as patients need to gain confidence in the equipment and become comfortable with the reactions of those who look at the device. At the end of the hospitalization, the patient must be able to walk alone and to climb the stairs. Exercise is stopped in case of subjective intolerance or drop in systolic pressure. Individuals are discharged home once they can mobilise independently, perform ADLs, climb stairs and have achieved a set level of knowledge regarding the device, so that they can care for themselves independently.

Physiotherapist must be trained in emergency procedures in case of device malfunctioning and must be also aware of patient hemodynamic instability and device dislodgement during mobilization. Exercise sessions are a good way also to teach the patient how to handle an alarm and a change of batteries.

\section{From 60-65 days to forever}

Out-patient to home-based rehabilitation [6,7] can be adopted. Exercise training (ET) program should be individualised to meet the patient's needs. The optimal time to start ET is yet to be defined: however, ET should be part of a multimodal rehabilitative intervention (including LVAD surveillance protocols, vital parameters monitoring, assessment of LVAD alarms, psychological support, nutritional encouragement and assistance, maximize patients' multidisciplinary recovery, carefully management orchestrated to empower recipients to care for themselves successfully in the community). ET should be safe and effective; heart rate (HR) and blood pressure (BP) assessment can be challenging patients with the continuous flow pumps, but the use of a Doppler BP monitor and appropriate sphygmomanometer, may assist in gaining more accurate readings. Maximal exercising HR and maximal exercise intensity may have been established by means of a cardiopulmonary exercise test prior to attendance at a CR programme and this information can be used to prescribe exercise intensity effectively. Individual's ability to perform at a prescribed intensity of exercise may be limited by the rate of the pump and the patient's underlying myocardial function. Subjective measures can also be used (RPE scale, changes in work of breathing and fatigue). Hypotension in low pump flows is one of the most common problems encountered, hence, the patient must be well educated regarding the normal flow values so that he/she is able to take appropriate action when required. Individuals may need encouragement to drink water before and during the exercise session to maintain pump flows. Moreover, the drive line insertion site will limit core muscle strength exercises and trunk range of movement. Movements restrictions are necessary also to prevent potential trauma of the drive line, moreover, bilateral arm exercise above the head should also be avoided due to increased stress on the abdominal musculature which can cause trauma to the drive-line. Finally, fast movements and ballistic type exercise should be avoided to reduce any micro-trauma at the drive-line site or damage to the drive-line itself.

Thus, each individual with a LVAD will require an individual assessment and exercise programme depending on their physical limitations and the ability of the device to affect exercise capacity. At the beginning of rehabilitative activities, some care must be given to the fact that the device could create an obstacle to physical recovery. We suggest that ET should be conducted with attention paid to patient's symptoms or clinical signs possibly indicating low peripheral perfusion. Gym sessions will include a warm up and cool down with conditioning and endurance exercises in between, and suitable exercises include 1) treadmill (increase incline, not speed), 2) static bike, 3) hamstring curls in standing position, 4) leg press, 5) bicep curls, 6) core stability, 7) respiratory muscle training. On the other hand, LVAD recipients should avoid 1) running on treadmill, 2) rowing machine, 3) cross trainer, 4) abdominals exercises, 5) bilateral arms above head with weights or abduction with weights, 6) swimming. ET should be eluded when: 1) VAD flow is different than "normal" flow rate, 2) systolic blood pressure is greater than $150 \mathrm{mmHg}$ or less than 80 $\mathrm{mmHg}$ in patients with pulsatile VADs, 3) mean arterial blood pressure is greater than 90 in patients with non-pulsatile VADs, 4) if fever is present, 5) if faint or headache are reported, 5) if short of breath, chest pain or thoracic pressure are complained. Standards of the increments of ET workloads during exercise sessions are lacking, but LVAD alarms and/or VAD-related problems should be considered as steps for increase the intensity or the frequency of exercise sessions. In Table 1 physical activity, according timing of implantation is provided. 
Table 1. Summary of functional recovery of LVAD, setting and monitoring, according to timing of device implantation.

\begin{tabular}{|c|c|c|c|c|c|}
\hline & $\begin{array}{l}\text { Management } \\
\text { setting }\end{array}$ & $\begin{array}{l}\text { Medical problems } \\
\text { and device } \\
\text { complications }\end{array}$ & $\begin{array}{l}\text { Vital signs, } \\
\text { ECG TLM } \\
\text { and device } \\
\text { parameters } \\
\text { monitoring }\end{array}$ & $\begin{array}{l}\text { Physical therapy } \\
\text { administered }\end{array}$ & $\begin{array}{l}\text { Responsible } \\
\text { for emergency } \\
\text { procedures }\end{array}$ \\
\hline From 0 to $10-15$ days & Post-acute care & +++ & +++ & $\begin{array}{l}\text { Safe sternal division, } \\
\text { heart chamber dissection, } \\
\text { respiratory function }\end{array}$ & $\begin{array}{l}\text { Post-acute care } \\
\text { physician and } \\
\text { LVAD coordinator }\end{array}$ \\
\hline From day $10 / 15$ to $30 / 35$ & In-patient rehabilitation & ++ & +++ & $\begin{array}{l}\text { Respiratory function } \\
\text { and EM }\end{array}$ & $\begin{array}{l}\text { LVAD coordinator } \\
\text { and physiotherapist }\end{array}$ \\
\hline From day $30 / 35$ to $60 / 65$ & Out-patient rehabilitation & + & ++ & $\mathrm{EM}+\mathrm{ET}$ & $\begin{array}{l}\text { Physiotherapist } \\
\text { (who refer to } \\
\text { LVAD coordinator) }\end{array}$ \\
\hline From day $60 / 65$ to forever & Home-based rehabilitation & + & + & ET & $\begin{array}{l}\text { Physiotherapist } \\
\text { Exercise therapist }\end{array}$ \\
\hline
\end{tabular}

LVAD, left ventricular assistance device; TLM, ECG-telemetry; EM, early mobilization; ET, exercise training.

Polastri et al. [10] should be congratulated, as they propose a series of supervised exercises in patients with a LVAD device, that are more prone to physical deconditioning due to forced bed rest and physical inactivity. EM should start as early as possible, and movements are selected to guarantee safety, involving all body segments, at the bedside, allowing wide choice of positions. They also confirm that duration, intensity and number of repetitions (frequency, and duration) of exercising is dependent on clinical conditions, medications given, LVAD working, peripheral myopathy and so on. They propose that physiotherapists are actively involved in the pathway of care. Drawbacks of Polastri et al. [10] manuscript are that EM might be interspaced by mechanical and medical LVAD complications and that functional recovery of LVAD recipients is a just part of the management of these patients. Early and late complications in LVAD patients [11] might interrupt the restoration program and LVAD recovery calls for a multi-disciplinary approach [6], with all health figures might help the LVAD recipients to return physically active and discharge safely in the community.

\section{References}

1. Go AS, Mozaffarian D, Roger VL, et al. Executive summary: Heart disease and stroke statistics-2013 Update: A report from the American Heart Association. Circulation 2013;127:143-52.

2. Hu C-S, Wu Q-H, Hu D-Y, Tkebuchava T. Treatment of chronic heart failure in the $21^{\text {st }}$ century: a new era of biomedical engineering has come. Chronic Dis Translat Med 2018 (in press). Doi: 10.1016/j.cdtm.2018.08.005

3. Kamdar F, John R, Eckman P, et al. Postcardiac transplant survival in the current era in patients receiving continuous-flow left ventricular assist devices. J Thorac Cardiovasc Surg 2013;145:575-81.
4. Elefteriades JA, Botta Jr DM. Avoiding technical pitfalls in Left Ventricular Assist Device placement. Cardiol Clin 2011;29;507-14.

5. Perme CS, Southard RE, Joyce DL, et al. Early mobilization of LVAD recipients: Who require prolonged mechanical ventilation. Tex Heart Inst J 2006;33:130-3.

6. Piepoli MF, Corrà U, Benzer W, et al. Cardiac Rehabilitation Section of the European Association of Cardiovascular Prevention and Rehabilitation. Secondary prevention through cardiac rehabilitation: From knowledge to implementation. A position paper from the Cardiac Rehabilitation Section of the European Association of Cardiovascular Preventionand Rehabilitation. Eur J Cardiovasc Prev Rehabil 2010; 17: 1-17.

7. Piepoli MF, Corrà U, Adamopoulos S, et al. Secondary prevention in the clinical management of patients with cardiovascular diseases. Core components, standards and outcome measures for referral and delivery: a policy statement from the cardiac rehabilitation section of the European Association for Cardiovascular Prevention \& Rehabilitation. Endorsed by the Committee for Practice Guidelines of the European Society of Cardiology. Eur J Prev Cardiol 2014;21:664-81.

8. Wells CL. Physical therapist management of patients with ventricular assist devices: key considerations for the acute care physical therapist. Phys Ther 2013;93:266-78.

9. Morrone TM, Buck LA, Catanese KA, et al. Early progressive mobilization of patients with left ventricular assist devices is safe and optimizes recovery before heart transplantation. J Heart Lung Transplant 1996;15:423-9.

10. Polastri M, Zagnoni G, Loforte A. Basic movements for postoperative exercise in patients with left ventricular assist devices. Monaldi Arch Chest Dis 2019;89:995.

11. Allen SJ, Sidebotham D. Postoperative care and complications after ventricular assist device implantation. Best Pract Res Clin Anaesthesiol 2012;26:231-46. 